\title{
Precision medicine and the antiphospholipid syndrome: what is the future?
}

\author{
Ignasi Rodriguez-Pintó ${ }^{1} \cdot$ Gerard Espinosa $^{2} \cdot$ Ricard Cervera $^{2}$ (I)
}

Received: 31 January 2020 / Revised: 31 January 2020 / Accepted: 7 February 2020 / Published online: 15 February 2020

(C) International League of Associations for Rheumatology (ILAR) 2020

The antiphospholipid syndrome (APS) is an autoimmune disorder characterized by the development of venous and/or arterial thrombosis, as well as obstetric morbidity (abortions, fetal deaths, and premature births), in the presence of antiphospholipid antibodies (aPL). The annual incidence of APS in adults is around 2 patients for 100,000 inhabitants and its prevalence around 50 cases per 100,000 inhabitants [1]. APS may be associated with other autoimmune diseases, mainly systemic lupus erythematosus (SLE), with a frequency of around $20-40 \%$, but it also develops in patients without any other identifiable autoimmune disease (primary APS). The 10year mortality rate in patients with APS is around 10\% [2].

APS was first described in 1983 when Graham R.V. Hughes and colleagues reported a high correlation between the development of thrombosis and the presence of a new antibody at that time, named anticardiolipin (aCL), as well as the presence of lupus anticoagulant (LAC). These findings led to the recognition of this new clinical syndrome called initially anticardiolipin syndrome and later renamed as APS. Since then, this disease has become one of the most common systemic autoimmune diseases and our knowledge of this condition has improved significantly. For instance, nowadays, we know that thrombotic events reappear in $30 \%$ of APS patients during the first 10 years after the diagnosis. The most common events are strokes, transient ischemic attacks, deep vein thromboses, and pulmonary embolisms, and among women who become pregnant, the most common obstetric complications are miscarriages [2]. Among those who had a successful pregnancy, $48 \%$ had premature births and $26 \%$ have intrauterine growth restriction [2]. A small subgroup of patients with

Ricard Cervera

rcervera@clinic.cat

1 Autoimmune Diseases Unit, Hospital Universitari Mútua de Terrassa, Terrassa, Catalonia, Spain

2 Department of Autoimmune Diseases, Hospital Clínic, University of Barcelona, Villarroel 170, 08036 Barcelona, Catalonia, Spain
APS (less than 1\%) suffered from a widespread coagulopathy leading to multiorgan failure, the so-named catastrophic APS (CAPS) [3].

The increased knowledge of APS highlighted the need for a clear definition of the disease across the disciplines and led to the proposal of a preliminary set of classification criteria in 1999 that were later modified in 2005. According to these criteria, APS is defined as the development of at least one clinical manifestation in patients that present at least one laboratory criterion [4]. Clinical criteria can be subdivided into thrombotic clinical manifestations where both venous and arterial thrombotic events are included and/or obstetric clinical manifestations that include three or more consecutive early pregnancy losses, a fetal loss or severe preeclampsia. The serological criteria require the presence of LAC, IgM, or $\mathrm{IgG}$ anticardiolipin $(\mathrm{aCL})$ at a titer higher than $40 \mathrm{UL} / \mathrm{ml}$ or IgM or IgG anti- $\beta 2$-glycoprotein I (aß2GPI) antibodies at a titer higher the 99th percentile twice over a 3-month period [4]. However, these classification criteria do not include widely accepted clinical manifestations of APS such as valvular heart disease, thrombocytopenia, APS nephropathy, livedo reticularis, or the longitudinal myelitis, neither newly developed aPL such as anti-phosphatidylserine/prothrombin antibodies (aPS/PT). An initiative to develop new classification criteria is currently ongoing.

Recent international guidelines recommend long-term anticoagulant treatment to any patient fulfilling definite APS and treatment with low-dose aspirin and heparin during the pregnancy to those women with a history of obstetric APS [5]. However, in the era of precision medicine, some further distinctions seem reasonable at the time to choose the treatment for APS patients. Some patients with aPL never develop any clinically significant problem while others develop thrombotic events or obstetric morbidity and a small proportion develop the catastrophic form.

Indeed, current and previous guidelines already subclassified asymptomatic aPL carriers in groups according to the risk of a thrombotic event based on their aPL profile $[5$, 
6]. The guidelines state that patients with persistent LAC, particularly if it is combined with the presence of aCL either IgG or IgM and a $\beta 2$ GPI either IgG or IgM (triple aPL positive) or have a medium-high levels, have a higher risk while those positive for only one antibody other than LAC at lowmedium titers have a low risk for future thrombosis or obstetric morbidity. In this sense, they propose to give treatment with low-dose aspirin as primary thromboprophylaxis to those patients with a high-risk profile or associated cardiovascular risk factors or SLE.

However, some controversies exist regarding the concept of triple positivity since some authors suggest that it might refer to the measurement of the same effect in three different ways. Further, some studies have shown conflicting results on the question of whether triple-positive aPL patients have a higher risk of clinical events than patients with positive LAC alone. Additionally, some studies did not find an association between IgM aCL and thrombosis and only LAC was found to be associated with adverse pregnancy outcomes [7].

Aiming to better predict thrombotic risk in patients with aPL, several risk scores have been proposed. In 2012, Otomo et al. [8] proposed and validated a risk score (called aPL-S), based on the presence and titers of several aPL. This score showed a moderate capacity to predict APS events with an area under the curve (AUC) of 0.75 . The authors found a value of 30 in aPL-S to be an independent factor for APS events from other traditional thrombotic risk factors. However, this score was later criticized by the insufficient capacity to predict events and the inclusion of some aPL that are not widely available in daily clinical practice. Furthermore, although the authors state that the score was found to have a hyperbolic pattern to predict pregnancy morbidity, this data was not shown nor further detailed, probably, because the cohort had a small number of obstetric events. Moreover, the score was not created to assess the other noncriteria manifestations of APS.

In 2013, Sciascia et al. [9] developed and validated a new risk score, called the Global APS Score (GAPSS). In this study, the authors included not only the aPL profile (LAC, aCL, aß2GPI, and aPS/PT) but also some conventional cardiovascular risk factors (hypertension and hyperlipydemia) and the use of thromboprophylaxis drugs. A GAPSS cut-off value of $\geq 10$ points showed the best diagnostic accuracy in the validation cohort and those patients that develop an arterial thrombosis were shown to have the highest score. Later, an upgraded version of GAPSS excluding anti-PS/PT was proposed. Initially, this score was derived from a SLE cohort; however, the last version of the score was later validated in patients with primary APS and in patients with APS associated with other autoimmune diseases [10-12].

Recently, a new score has been proposed to predict the thrombotic risk in SLE patients [12]. The authors included the presence of LAC (or anti-PS/PT in their absence), low
C3, and C4d deposited on platelets. This score is based on a retrospective review of a 5-year history of thrombosis. The authors showed that the score had an Akaike information criterion (AIC) of 79 to predict the event and an AIC of 85 when the aPS/PT were used. Additionally, the results showed that the combination of low $\mathrm{C} 3$ and aPS/PT positivity had an AIC of 90 to predict a history of thrombosis in SLE patients [12]. Obviously, a prospective study is needed to confirm these findings since the derivation cohort was the same as the validation cohort. Furthermore, it needs to be confirmed in patients without SLE (i.e., asymptomatic aPL carriers and primary APS patients), as well as in patients from different ethnic origins.

Since the first description of APS in 1983, a long way has already been overcome. However, new exciting knowledge is coming up in this field since the wider awareness of this disease has increased its interest. It seems clear that the identification of subgroups of patients with distinct clinical features will allow a more precise therapeutic strategy and better patients care. However, the best clustering strategy is still a matter of debate. Probably, several other underappreciated factors might influence the risk of moving the balance to or away the thrombotic state. It is still needed to clarify if the future integration of artificial intelligence and big data to patient care might help in the management of the patients with APS considering hundreds of other risk factors unappreciated until now.

\section{Compliance with ethical standards}

Conflict of interest disclosure statement The authors have no conflict of interest.

\section{References}

1. Duarte-García A, Pham MM, Crowson CS, Amin S, Moder KG, Pruthi RK, Warrington KJ, Matteson EL (2019) The epidemiology of antiphospholipid syndrome: a population-based study. Arthritis Rheum. 71(9):1545-1552

2. Cervera R, Serrano R, Pons-Estel GJ et al (2015) Morbidity and mortality in the antiphospholipid syndrome during a 10 -year period: a multicentre prospective study of 1000 patients. Ann. Rheum. Dis. 74(6):1011-1018 http://www.ncbi.nlm.nih.gov/pubmed/ 24464962.

3. Asherson RA (1992) The catastrophic antiphospholipid syndrome. J. Rheumatol. 19(4):508-512 http://www.ncbi.nlm.nih.gov/ pubmed/1593568.

4. Miyakis S, Lockshin MD, Atsumi T et al (2006) International consensus statement on an update of the classification criteria for definite antiphospholipid syndrome (APS). J. Thromb. Haemost. : JTH 4(2):295-306 http://www.ncbi.nlm.nih.gov/pubmed/16420554.

5. Tektonidou MG, Andreoli L, Limper M, Amoura Z, Cervera R, Costedoat-Chalumeau N, Cuadrado MJ, Dörner T, Ferrer-Oliveras R, Hambly K, Khamashta MA, King J, Marchiori F, Meroni PL, Mosca M, Pengo V, Raio L, Ruiz-Irastorza G, Shoenfeld Y, Stojanovich L, Svenungsson E, Wahl D, Tincani A, Ward MM 
(2019) EULAR recommendations for the management of antiphospholipid syndrome in adults. Ann Rheum Dis 78(10): 1296-1304

6. Ruiz-Irastorza G, Cuadrado M, Ruiz-Arruza I et al (2011) Evidence-based recommendations for the prevention and longterm management of thrombosis in antiphospholipid antibodypositive patients: report of a Task Force at the 13th International Congress on Antiphospholipid Antibodies. Lupus 20(2):206-218 http://www.ncbi.nlm.nih.gov/pubmed/21303837.

7. Yelnik CM, Laskin CA, Porter TF et al (2016) Lupus anticoagulant is the main predictor of adverse pregnancy outcomes in aPLpositive patients: validation of PROMISSE study results. Lupus Sci Med 3:1

8. Otomo K, Atsumi T, Amengual O et al (2012) Efficacy of the antiphospholipid score for the diagnosis of antiphospholipid syndrome and its predictive value for thrombotic events. Arthritis Rheum 64(2):504-512

9. Sciascia S, Sanna G, Murru V, Roccatello D, Khamashta MA, Bertolaccini ML (2013) GAPSS: the Global Anti-Phospholipid
Syndrome Score. Rheumatology (Oxford, England) 52(8):13971403 http://www.ncbi.nlm.nih.gov/pubmed/23315788.

10. Radin M, Schreiber K, Costanzo P et al (2017) The adjusted Global AntiphosPholipid Syndrome Score (aGAPSS) for risk stratification in young APS patients with acute myocardial infarction. Int $\mathrm{J}$ Cardiol 240:72-77. https://doi.org/10.1016/j.ijcard.2017.02.155.

12. Fernandez Mosteirin N, Saez Comet L, Salvador Osuna C, Calvo Villas JM, Velilla MJ (2017) Independent validation of the adjusted GAPSS: role of thrombotic risk assessment in the real-life setting. Lupus 26(12):1328-1332

13. Petri MA, Conklin J, O'malley T, Dervieux T (2019) Platelet-bound $\mathrm{C} 4 \mathrm{~d}$, low $\mathrm{C} 3$ and lupus anticoagulant associate with thrombosis in SLE. Lupus Sci Med 6:318 http://lupus.bmj.com/.

Publisher's note Springer Nature remains neutral with regard to jurisdictional claims in published maps and institutional affiliations. 